\title{
SUTURELESS THYROIDECTOMY WITH BIPOLAR DIATHERMY
}

\author{
Ravi Kumar $H^{1}$, Sunil Kumar S. R2, Siddharth Gosavi ${ }^{3}$ \\ ${ }^{1}$ Associate Professor, Department of General Surgery, Vydehi Institute of Medical Sciences and Research Centre, Bengaluru. \\ ${ }^{2}$ Assistant Professor, Department of General Surgery, Vydehi Institute of Medical Sciences and Research Centre, Bengaluru. \\ ${ }^{3}$ Intern Medical Officer, Department of General Surgery, Vydehi Institute of Medical Sciences and Research Centre, Bengaluru.
}

\section{ABSTRACT}

The thyroid gland is one of the most vascular organs in the body and surgical resection mandates meticulous surgical technique and haemostasis. The rate of complications of thyroid surgeries were very high earlier but with the advent of ligasure, harmonic scalpel and bipolar surgical diathermy complications have reduced. In this study, we have used bipolar surgical diathermy.

\section{MATERIALS}

116 patients with thyroid swelling attending surgical OPD in Vydehi Institute of Medical Sciences and Research Centre during the period of 2011-2015 were included in the study. Proper preoperative workup was done and sutureless thyroidectomy surgeries were done using bipolar surgical diathermy. Patients were reviewed for any complications following thyroidectomy like haemorrhage, hypocalcaemia and recurrent laryngeal nerve palsy.

\section{RESULTS}

Out of 116 patients who underwent sutureless thyroidectomy none of them had any primary, secondary or reactionary haemorrhage, one patient who underwent total thyroidectomy for follicular carcinoma had transient hypocalcaemia.

\section{CONCLUSION}

Sutureless thyroidectomy is a very good surgical practice with bipolar surgical diathermy and it permits low postoperative complications and cost effectiveness.

\section{KEYWORDS}

Sutureless Thyroidectomy, Bipolar Diathermy.

HOW TO CITE THIS ARTICLE: Kumar RH, Kumar SSR, Gosavi S. Sutureless thyroidectomy with bipolar diathermy. J. Evolution Med. Dent. Sci. 2016;5(42):2574-2577, DOI: 10.14260/jemds/2016/602

\section{INTRODUCTION}

The thyroid gland is one of the most vascular organs in the body. The adult thyroid gland is brown in colour and firm in consistency and is located posterior to the strap muscles. The normal thyroid gland weighs approximately $20 \mathrm{~g}$, but gland weight varies with body weight and iodine intake. The thyroid lobes are located adjacent to the thyroid cartilage and connected in the midline by an isthmus that is located just inferior to the cricoid cartilage. A pyramidal lobe is present in about $50 \%$ of patients. The thyroid lobes extend to the midthyroid cartilage superiorly and lie adjacent to the carotid sheaths and sternocleidomastoid muscles laterally. The strap muscles (Sternohyoid, sternothyroid and superior belly of the omohyoid) are located anteriorly and are innervated by the ansa cervicalis (Ansa hypoglossi). The thyroid gland is enveloped by a loosely connecting fascia that is formed from the partition of the deep cervical fascia into anterior and posterior divisions. The true capsule of the thyroid is a thin, densely adherent fibrous layer that sends out septa that invaginate into the gland, forming pseudolobules. The thyroid capsule is condensed into the posterior suspensory or Berry's ligament near the cricoid cartilage and upper tracheal rings. Microscopically, the thyroid is divided into lobules that

Financial or Other, Competing Interest: None.

Submission 08-04-2016, Peer Review 04-05-2016,

Acceptance 11-05-2016, Published 24-05-2016.

Corresponding Author:

Dr. Ravi Kumar $H$,

No. 28 M, Gandhinagar, Munekola,

Marathahalli Post, Bangalore-560037.

E-mail:drravikumar2000@yahoo.com

DOI: $10.14260 /$ jemds/2016/602 contain 20 to 40 follicles. There are about $3 \times 106$ follicles in the adult male thyroid gland. The follicles are spherical and average $30 \mathrm{~m}$ in diameter. Each follicle is lined by cuboidal epithelial cells and contains a central store of colloid secreted from the epithelial cells under the influence of the pituitary hormone TSH. The second group of thyroid secretory cells is the $\mathrm{C}$ cells or parafollicular cells, which contain and secrete the hormone calcitonin. They are found as individual cells or clumped in small groups in the interfollicular stroma and located in the upper poles of the thyroid lobes. Free thyroid hormone enters the cell membrane by diffusion or by specific carriers and is carried to the nuclear membrane by binding to specific proteins. $\mathrm{T} 4$ is deiodinated to $\mathrm{T} 3$ and enters the nucleus via active transport, where it binds to the thyroid hormone receptor. The $\mathrm{T} 3$ receptor is similar to the nuclear receptors for glucocorticoids, mineralocorticoids, oestrogens, vitamin $\mathrm{D}$ and retinoic acid. In humans, two types of T3 receptor genes are located on chromosomes 3 and 17. Thyroid receptor expression depends upon peripheral concentrations of thyroid hormones and its tissue specific form is abundant in the central nervous system, whereas the form predominates in the liver. Each gene product has a ligand-independent, aminoterminal domain; a ligand-binding, carboxy-terminal domain; and centrally located DNA-binding regions. Binding of thyroid hormone leads to the transcription and translation of specific hormone responsive genes. Thyroid hormones affect almost every system in the body. They are important for foetal brain development and skeletal maturation. T3 increases oxygen consumption, basal metabolic rate and heat production by stimulation of $\mathrm{Na}+\mathrm{K}+$ ATPase in various tissues. It also has positive inotropic and chronotropic effects on the heart by increasing transcription of the $\mathrm{Ca} 2+$ ATPase in the 
sarcoplasmic reticulum and increasing levels of betaadrenergic receptors and concentration of $\mathrm{G}$ proteins. Myocardial alpha receptors are decreased and actions of catecholamines are amplified. Thyroid hormones are responsible for maintaining the normal hypoxic and hypercapnic drive in the respiratory center of the brain. They also increase GI motility, leading to diarrhoea in hyperthyroidism and constipation in hypothyroidism. Thyroid hormones also increase bone and protein turnover and the speed of muscle contraction and relaxation. They also increase glycogenolysis, hepatic gluconeogenesis, intestinal glucose absorption and cholesterol synthesis and degradation. In 1776 , the thyroid was classified as a ductless gland by Albrecht von Haller and was thought to have numerous functions ranging from lubrication of the larynx to acting as a reservoir for blood to provide continuous flow to the brain. The most notable thyroid surgeons were Emil Theodor Kocher (18411917) and C.A. Theodor Billroth (1829-1894), who performed thousands of operations with increasingly successful results. However, as more patients survived thyroid operations, new problems and issues became apparent. After total thyroidectomy, patients (particularly children) became myxedematous with cretinous features. Myxoedema was first effectively treated in 1891 by George Murray using a subcutaneous injection of an extract of sheep's thyroid and later, Edward Fox demonstrated that oral therapy was equally effective. In 1909, Kocher was awarded the Nobel Prize for medicine in recognition for his work on the physiology, pathology and surgery of the thyroid gland. Early in the history of thyroid surgery, the pioneers of endocrine surgery such as Billroth and Kocher experienced greater than 50\% mortality due to bleeding complications. Nerves, parathyroids and surrounding structures are all at risk of injury during thyroidectomy. Injury to the RLN may occur by severance, ligation or traction, but should occur in $<1 \%$ of patients undergoing thyroidectomy by experienced surgeons. The RLN is most vulnerable to injury during the last 2 to $3 \mathrm{~cm}$ of its course, but also can be damaged if the surgeon is not alert to the possibility of nerve branches and the presence of a nonrecurrent nerve, particularly on the right side. If the injury is recognized intraoperatively, most surgeons advocate primary reapproximation of the perineurium using non-absorbable sutures. Approximately, $20 \%$ of patients are at risk of injury to the external branches of the superior laryngeal nerve, especially if superior pole vessels are ligated en masse. Intraoperative RLN and external laryngeal nerve monitoring techniques are being increasingly used during thyroid and parathyroid surgery. Both continuous monitoring using endotracheal tube electrodes and intermittent monitoring by periodic stimulation and laryngeal palpation are used. No large scale studies have shown that nerve monitoring equivocally reduces nerve injury, particularly by experienced surgeons. The cervical sympathetic trunk is at risk of injury in invasive thyroid cancers and retro-oesophageal goitres and may result in Horner's syndrome. Transient hypocalcaemia has been reported in up to $50 \%$ of cases, but permanent hypoparathyroidism occurs $<2 \%$ of the time. Postoperative hypocalcaemia is more likely in patients who undergo concomitant thyroidectomy, central and lateral neck dissection. Postoperative haematomas or bleeding may also complicate thyroidectomies and rarely necessitate emergency reoperation to evacuate the haematoma. Bilateral vocal cord dysfunction with airway compromises requires immediate reintubation and tracheostomy. Seromas may need aspiration to relieve patient discomfort. Wound cellulitis and infection and injury to surrounding structures such as the carotid artery, jugular vein and oesophagus are infrequent. ${ }^{1}$ Since that time, the technique of thyroidectomy has evolved to a safe and haemostatic operation.2,3 Recurrent laryngeal nerve injury after total thyroidectomy is a dreaded complication. Most of the reports in literature suggest that the rate of transient and permanent vocal cord immobility after thyroid surgery is 4$7 \%$ and $1-4 \%$. Rates of complications and morbidity in thyroid surgery have decreased greatly because of new technologies that are being invented like ligasure, harmonic scalpel and bipolar surgical diathermy. During total thyroidectomy, it is reported that failure to identify recurrent laryngeal nerve is 5$18 \%$; routine identification of recurrent laryngeal nerve during thyroid surgery has reduced the injury rate from $10 \%$ to $4 \%{ }^{4}$

Most of the studies have been performed comparing ligasure and suture knot tying or harmonic scalpel with conventional knot tying. In this study, we chose to use bipolar electrocautery for performing thyroid surgeries that is sutureless thyroidectomy.

\section{MATERIALS AND METHODS}

116 patients with thyroid swelling attending surgical OPD in Vydehi Institute of Medical Sciences and Research Centre during the period of 2011-2015 were included in the study. Among the 116 patients, 52 cases were diagnosed with solitary thyroid nodule, 30 cases were diagnosed with multinodular goitre, 17 cases were diagnosed with carcinoma thyroid and 17 cases were diagnosed with Grave's disease. All patients underwent standard evaluation for thyroid surgery including one or more of the following as indicated on a case by case basis: routine blood investigations including thyroid function tests, fine needle aspiration, ultrasonography and computed tomography of the neck and chest and indirect laryngoscopy was performed. The study was commenced after approval from the Vydehi Institute of Medical Sciences and Research Centre Institutional Ethics Committee and is in accordance with the Declaration of Helsinki of 1975 and revised in 2000. Written, informed and understood consent was obtained from the patients before starting the study. Patients also gave consent for the data to be reported and published.

After endotracheal intubation under general anaesthesia, patient was positioned in reverse Trendelenburg position. Kocher's neck incision was placed and extended from one sternocleidomastoid to other. Upper and lower flaps were raised up till thyroid notch and suprasternal notch. Deep fascia was incised vertically in midline. Strap muscles were cut at the junction of upper $1 / 3^{\text {rd }}$ and lower $2 / 3^{\text {rd }}$ border and retracted depending on the size of the gland; 2 tributaries of middle thyroid vein were identified and cauterized with bipolar electrothermal cautery. Superior thyroid pedicle was dissected. All terminal branches of superior thyroid artery, inferior thyroid artery and other unnamed vessels were cauterized with bipolar cautery. Recurrent laryngeal nerve was identified on both the sides and preserved and parathyroid glands were identified at least on one side and preserved in all possible conditions. Proper haemostasis was achieved. Romovac suction drain kept in all cases. Strap muscles and deep fascia were sutured with vicryl; platysma 
was sutured with vicryl. Skin margins were approximated with monocryl. Suction drain was removed after 48 hours; patients were given broad-spectrum antibiotics for 2 days and most patients were discharged on 3rd postoperative day.

Follow-up of the patients was done after 10 days, 6 weeks and 12 weeks for any recurrent laryngeal nerve palsy, hypocalcaemia.

\section{RESULTS}

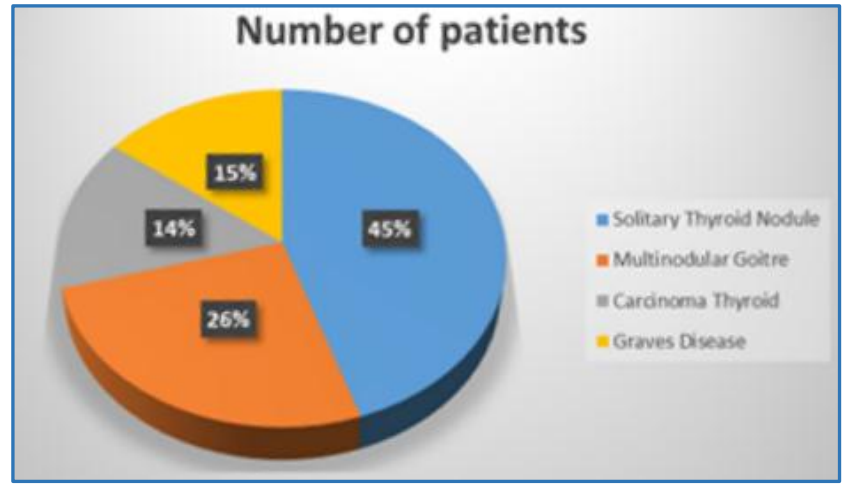

Fig. 1: Distribution of Thyroid Disorders for which Thyroidectomy was Done

\begin{tabular}{|c|c|}
\hline Thyroid Disorder & Number of Patients \\
\hline Solitary Thyroid Nodule & 52 \\
\hline Multinodular Goitre & 30 \\
\hline Carcinoma Thyroid & 17 \\
\hline Graves' Disease & 17 \\
\hline \multicolumn{2}{|c|}{ Table 1 } \\
\hline
\end{tabular}

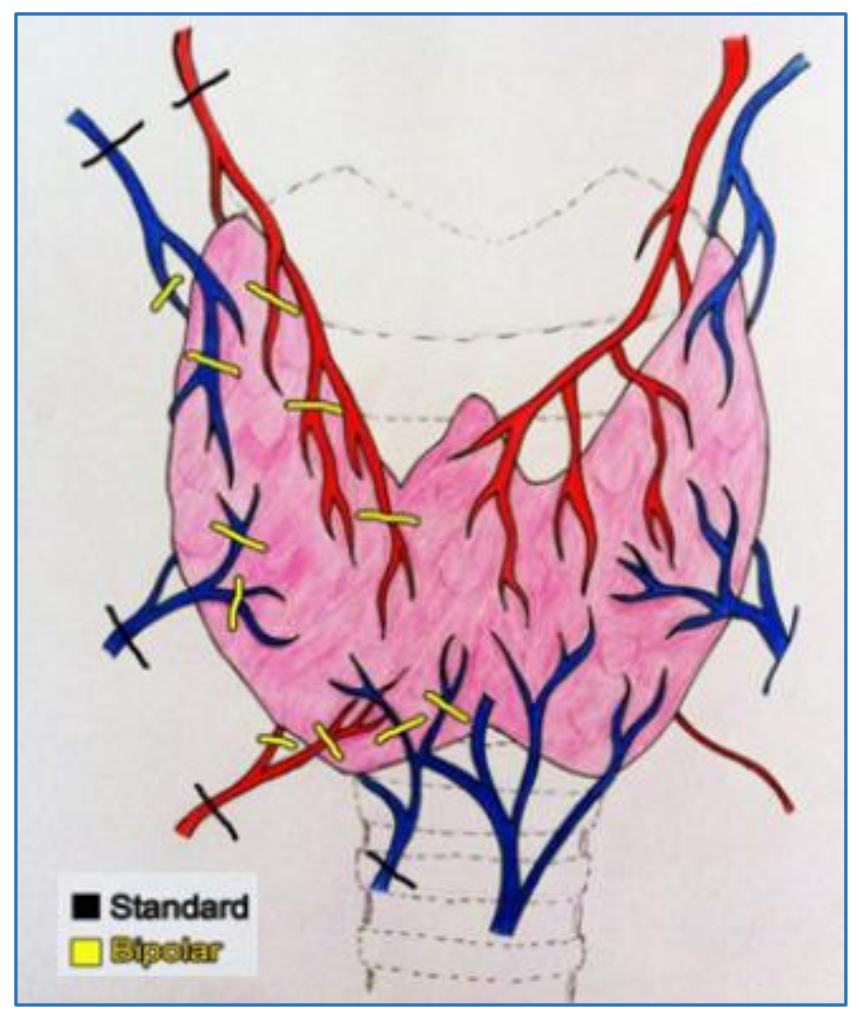

Fig. 2: Schematic Diagram showing Difference between Standard Knot Tying and Bipolar Surgical Diathermy. ${ }^{4}$

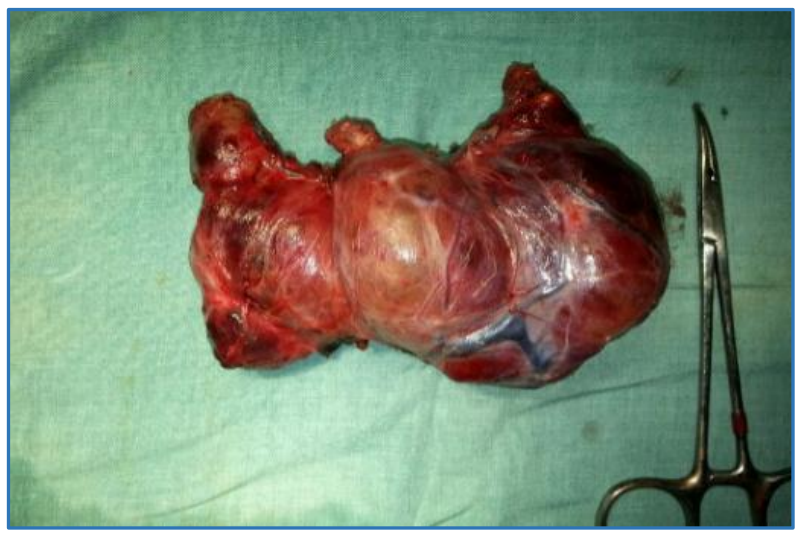

Fig. 3: Thyroid Specimen after Thyroidectomy with Lobes and Isthmus

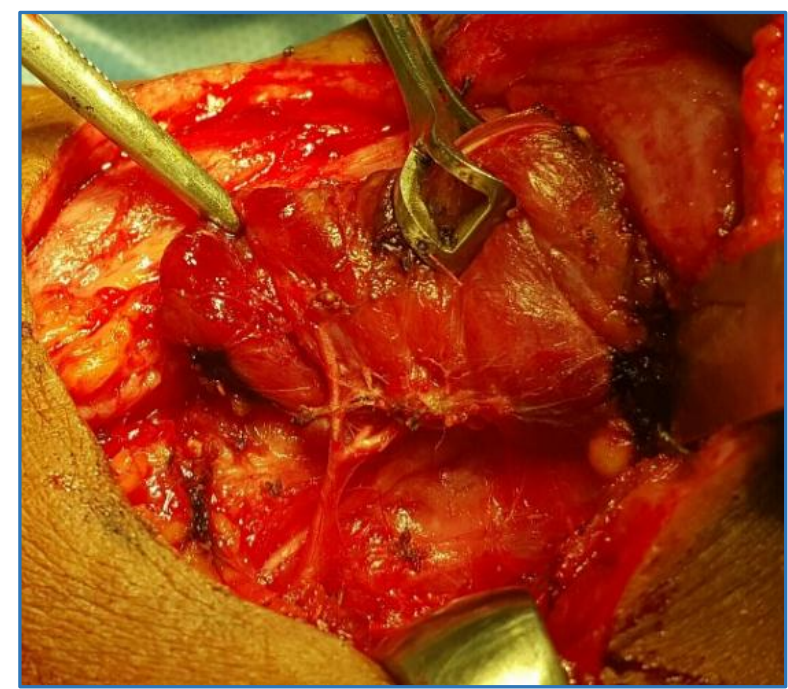

Fig. 4: Inferior Thyroid Artery and Recurrent Laryngeal Nerve

Out of 116 patients who underwent sutureless thyroidectomy, none of them had any primary, secondary or reactionary haemorrhage. One patient who underwent total thyroidectomy for follicular carcinoma had transient hypocalcaemia and was treated with Vitamin D, calcium gluconate and patient recovered in 2 weeks.

\section{DISCUSSION}

Thyroid surgery can be performed safely with the appropriate technique, meticulous haemostasis and precise knowledge of the anatomy. ${ }^{5}$ Bleeding is a major complication of thyroid surgery, both intraoperative and postoperative with the potential to cause life-threatening airway obstruction, haemodynamic shock and even death. Sometimes, it requires reoperation and may prolong the operation and stay. During thyroidectomy, bleeding can obscure the operative field making safe dissection of recurrent laryngeal nerve and parathyroid gland difficult and prolonging the operation time.

Effective vessel haemostasis can be achieved by suture knot tying technique or newer techniques of vessel haemostasis like harmonic scalpel, ligasure (Alternative bipolar surgical diathermy system), clips, staples, tissue adhesives, haemostatic agents/sealants, monopolar and bipolar surgical diathermy. The safest, most efficient and cost effective way to achieve haemostasis is still debated a lot. During the last decade, alternative techniques for improving 
safety and effectiveness of thyroidectomy have been developed. The conventional suture knot tying technique requires a large number of surgical ties and is time consuming, decreases healing as well as increases wound infection, requires good exposure, injury to neighbouring structures and foreign body reaction. ${ }^{6}$ Harmonic scalpel uses ultrasound technology to denature protein in the vessel wall and tissues up till $5 \mathrm{~mm}$ thickness leading to coagulation. ${ }^{7}$ A major criticism to harmonic scalpel comes from its cost; it is disposable and expensive. ${ }^{8}$ Ligasure vessel sealing device utilizes both electrical and elastin in vessel walls and tissue up till $7 \mathrm{~mm}$ in diameter to provide haemostasis. ${ }^{9}$ Use of the ligasure in thyroid surgery did not significantly reduce operative time, blood loss or complication rates compared with conventional knot tying but it increased operative cost.10

A monopolar diathermy can be applied quickly and safely to vessels $<2 \mathrm{~mm}$ in diameter, but the extension of the lateral thermal energy it produces cannot be estimated precisely, so it can cause inadvertent thermal injury to adjacent structures. $^{11}$ An alternative to conventional surgical diathermy is bipolar surgical diathermy. The path of the current can be constrained to pass only through the tissues being treated. This is achieved using a special forceps in which the two halves of the instrument are insulated from one another and in effect one half becomes the source of current and the other destination. A small blood vessel gripped between the jaws will be coagulated, whereas there will be no damage to the adjacent structures; most of the modern diathermy machines support the use of bipolar probe without modification unlike ligasure and harmonic scalpel in which the machine is very expensive.

\section{CONCLUSION}

Sutureless thyroidectomy is a very good surgical practice with bipolar surgical diathermy under experienced surgeon and it permits low postoperative complications and cost effectiveness.

\section{REFERENCES}

1. Geeta Lal, Orlo H Clark. Thyroid parathyroid and adrenal. Schwartz's principles of surgery. McGraw Hill Companies Inc Chapter 38, 2010; $9^{\text {th }}$ edn:p 1344.

2. Ming-Lang Shih, Quan-Yang Duh, Chung-Bao Hsieh, et al. Bilateral superficial cervical plexus block combined with general anaesthesia administered in thyroid operations. World Journal of Surgery 2010;34(10):2338-43.

3. Jeffrey C Liu, Jatin P Shah. Surgical technique refinements in head and neck oncologic surgery. Journal of Surgical Oncology 2010;101(8):661-8.

4. Domenico Parmeggiani, Massimo De Falco, Nicola Avenia, et al. Nerve sparing sutureless total thyroidectomy. Ann Ital Chir 2011;1-2.

5. Sreenivasa Rao, Sushama Surapaneni. Sutureless thyroidectomy. online document 2015;1-5. www.jemds.com/data_pdf/Sutureless $\% 20$ thyroidectom y.docx, accessed on November $1^{\text {st. }}$.

6. Vesely DL, Angtuaco EJ, Boyd CM. Sinus tract in the neck: a rare complication of subtotal thyroidectomy for graves disease. J Med 1986;17(3-4):253-61.

7. Ethicon Harmonic Focus. http://www.harmonic.com/dtcf/pages/HarmonicFocus. htm. Accessed 10th November, 2015.

8. Emanuele Ferri, Enrico Armato, Giacomo Spinato, et al. Focus harmonic scalpel compared to conventional haemostasis in open total thyroidectomy: a prospective randomized trial. International Journal of Otolaryngology Article ID 357195, 2011;p 7. doi: 10.1155/2011/357195

9. Valley Lab. Technology. http://www.ligasure.com/ligasure/pages.aspx. Accessed $10^{\text {th }}$ November, 2015.

10. Kiriakopoulos A, Dimitrios T, Dimitrios L. Use of a diathermy system in thyroid surgery. Arch Surg 2004;139(9):997-1000.

11. Sengupta $S$, Webb DR. Use of a computer-controlled bipolar surgical diathermy system in radical prostatectomies and other open urological surgery. ANZ J Surg 2001;71(9):538-40. 